\title{
Los visitantes en los museos de arte argentinos: consideraciones a partir de los estudios de visitantes/públicos
}

\section{Artículo de reflexión}

\section{Alejandra Gabriela Panozzo Zenere}

CONICET - Universidad Nacional de Rosario, Argentina panozzo.a@gmail.com

Recibido: 24 de marzo de 2018

Aprobado: 25 de abril de 2018

Cómo citar este artículo: Panozzo Zenere, Alejandra Gabriela (2019). Los visitantes en los museos de arte argentinos: consideraciones a partir de los estudios de visitantes/públicos. Calle 14: revista de investigación en el campo del arte 14(25), pp. 58-71. DOI: https://doi. org/10.14483/21450706.14048

\section{(2) (1)}

https://creativecommons.org/licenses/by/4.0/deed.es 


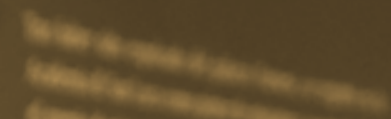

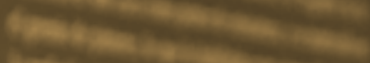

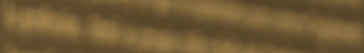

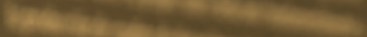

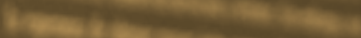

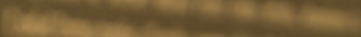
track

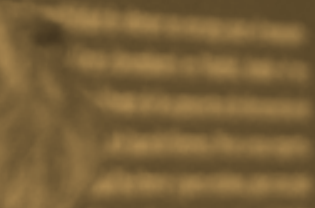

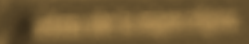

(1) 9.15
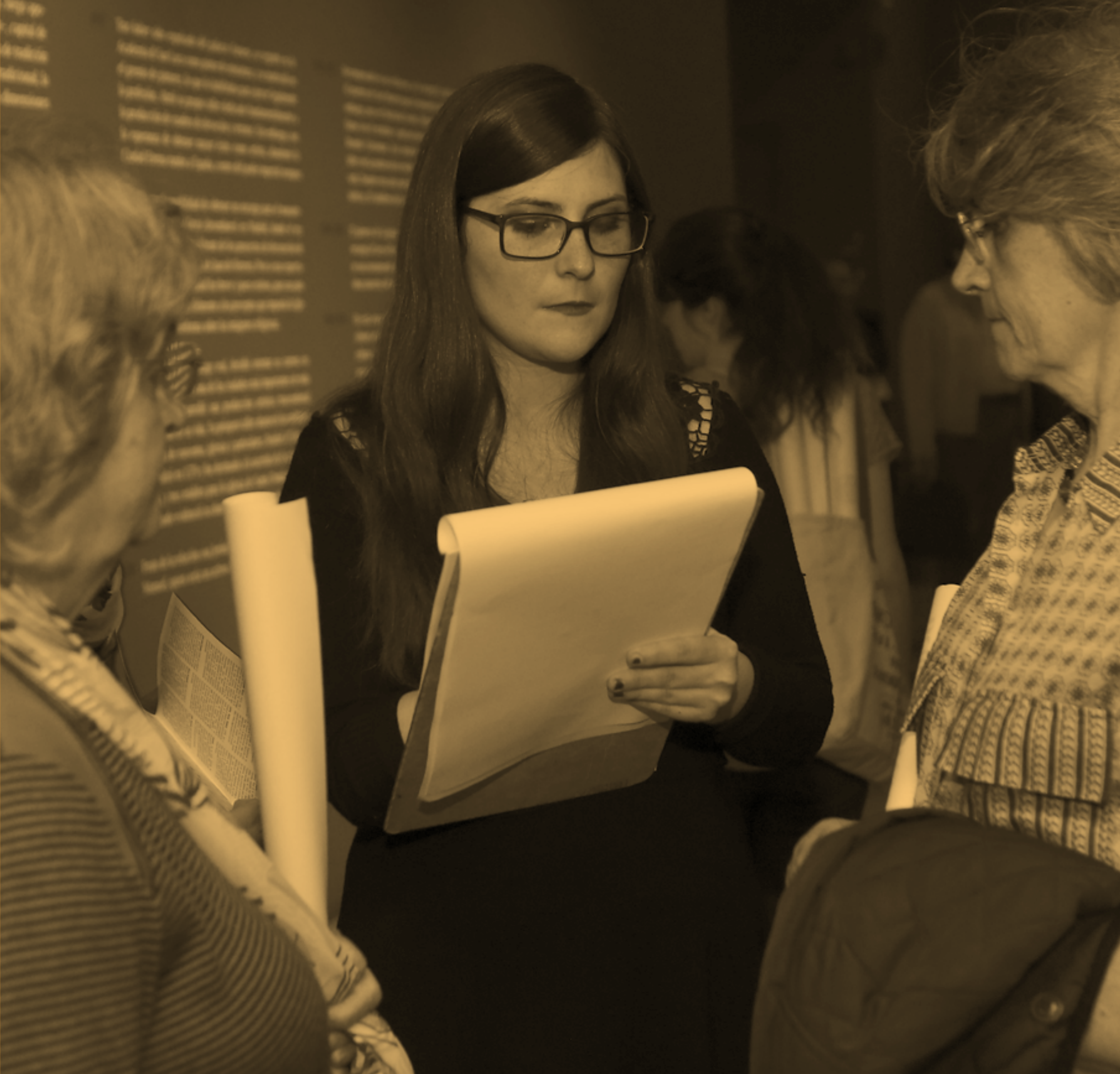


\title{
Resumen
}

El modelo museístico contemporáneo establece una lógica de disciplinamiento vinculada a múltiples ofertas de experiencias, de entretenimiento, de consumo y de diálogo. En este esquema, los gustos, los intereses y las necesidades de los visitantes se vuelven fundamentales para que el museo se reconfigure ante los desafíos del sistema capitalista. La discusión del presente texto abordará las diferencias que presentan los museos de arte argentinos al generar instancias de indagación sobre sus públicos. Para ello, el trabajo procura desentrañar el nuevo vínculo que se establece con los visitantes desde un contexto determinado y una tipología específica, lo que ayuda a comprender la dinámica que adquieren estos establecimientos en la contemporaneidad. En líneas generales, se apela a un cruce de contenidos sustentados en los enfoques teórico-críticos aplicados a la museología y los estudios de visitantes y, específicamente, se aportan datos analíticos sobre la utilización de los estudios de visitantes en los museos de arte argentinos. Además, se recurre al estudio de casos múltiples al describir algunos estudios que permiten reconocer instancias de diálogo en la dinámica museal.

\section{Palabras claves}

Museos de arte argentinos; experiencia; pasivo-activo; estudios de visitantes; cuantitativocualitativo

\begin{abstract}
The contemporary museum model establishes a logic of discipline linked to multiple offers of experiences, entertainment, consumption and dialogue. In this scheme, the tastes, interests and needs of the visitors become fundamental for the museum to reconfigure itself before the challenges of the capitalist system. This article will address the quandaries faced by Argentine art museums when they generate instances of inquiry about their audiences. To do this, we seek to unravel the new link that is established with visitors from a specific context and a specific typology, an aid to understand the dynamics that these establishments exhibit today. More generally, we appeal to the crossing of contents based on critico-theoretical approaches applied to museology and visitor studies and, specifically, analytical data is provided on the use of visitor's studies in Argentine art museums. In addition, multiple cases are used when describing studies that allow the recognition of instances of dialogue in the museal dynamics.
\end{abstract}

\section{Keywords}

Argentine art museums; experience; passive-active; visitor studies; quantitative-qualitative

\section{Résumé}

Le modèle de musée contemporain instaure une logique de discipline liée à de multiples offres d'expériences, de divertissement, de consommation et de dialogue. Dans ce schéma, les goûts, les intérêts et les besoins des visiteurs deviennent fondamentaux pour que le musée se reconfigure face aux défis du système capitaliste. Cet article abordera les dilemmes auxquels les musées d'art argentins sont confrontés lorsqu'ils suscitent des enquêtes sur leurs publics. Pour ce faire, nous cherchons à démêler le nouveau lien établi avec les visiteurs dans un contexte et une typologie spécifiques, ce qui aide à comprendre la dynamique que ces établissements présentent aujourd'hui. De manière plus générale, nous faisons appel au croisement de contenus basé sur des approches théorico-critiques appliquées à la muséologie et aux études sur les 
visiteurs. Des données analytiques sont notamment fournies sur l'utilisation des études de visiteurs dans les musées d'art argentins. De plus, plusieurs cas sont utilisés pour décrire des études permettant la reconnaissance d'instances de dialogue dans la dynamique muséale.

Mots clés

Musées d'art argentin ; expérience ; passif-actif ; études de visiteurs ; quantitatif qualitatif

Visitantes de museus de arte argentinos: considerações baseadas em estudos de visitantes/públicos

\section{Resumo}

O modelo de museu contemporâneo estabelece uma lógica de disciplina ligada a múltiplas ofertas de experiências, entretenimento, consumo e diálogo. Neste esquema, os gostos, interesses e necessidades dos visitantes tornam-se fundamentais para o museu se reconfigurar diante dos desafios do sistema capitalista. Este artigo abordará os dilemas enfrentados pelos museus argentinos de arte quando geram instâncias de investigação sobre seus públicos. Para isso, buscamos desvendar o novo elo que é estabelecido com os visitantes de um contexto específico e uma tipologia específica, o que ajuda a entender a dinâmica que esses estabelecimentos exibem hoje. De maneira mais geral, apelamos ao cruzamento de conteúdos baseados em abordagens teórico-críticas aplicadas à museologia e aos estudos de visitantes e, especificamente, dados analíticos sobre o uso de estudos de visitantes em museus de arte argentinos. Além disso, o estudo de casos múltiplos é utilizado na descrição de estudos que permitem o reconhecimento de instâncias de diálogo na dinâmica museal.

\section{Palavras-chaves}

Museus de arte argentinos; experiência; passivo-ativo; estudos de visitantes; quantitativoqualitativo

Sug chaiagkasina chi tsabajukuna kawangapa rirkakuna chi Argintinu ruraskata: kawarkakuna imasami maipipuna chi iachachiipi hi chaigkuna kawarkakuna chi puglupi

\section{Maillallachiska}

Kai tsabaja kai punchakuna ruraska kami iapa mucuchu i chasaiatata iamasami nukanchpa antiwa iachachirirka, parlanakui, gastaikuna i rimanakui. Kai kawaikuna, imami munakuna imami kurtinta i imami kai ruragkuna munankuna chi sug luarmandakunawa iapa iukar kulki kagkunawa.

Kaiparlanakui imasami ruraska, kaiarimi imasa iakichinakui tiaska kikin pura kai tsabaju Argentinukuna ruraska. Chasapa ikuti kaiarinkuna sugrgcha tsabaju rurai chasa aidami intindingapa imasami kai punchakuna chi tsabajukunaka rurai. Chasa ruraspaka sugama sugma rimanakuspa. Minkunami imami chaia rurangapa mana tsukai tiangapa kai chaiamigkuna kai musiu, kai aldami iuiaringapa imasami iuiarirka imasami iachaikurkakuna chi tsabaju Argentikui. Chasa kai iachaikaipi kawachirirka chi musé tsabajukuna ruraskata.

\section{Rimangapa Ministidukuna}

Tsabajukuna kawachii aregentinukunapa; iachikuikuna; aidachinakuspa aichaikuikuna kai chaiagkunapa; kuintsururai-kawachingapa iatata 


\section{I.}

El museo es un instrumento del sistema capitalista, por tanto, su desarrollo depende de sus propias variaciones y las del sistema (Bennett, 1996). Los museos de arte reflejan esta afirmación al tratarse de la entidad más floreciente y compleja en su estructura constitutiva (Duncan y Wallach, 1978; 1980; Duncan, 1995). A raíz de ello, los distintos modelos surgidos a lo largo de su historia ${ }^{1}$ se ven en la necesidad de adaptarse a las nuevas lógicas con que opera el arquetipo contemporáneo. El principal rasgo que detectamos dentro de las prácticas que adquiere este modelo es sin duda, el desplazamiento del centro de atención de los objetos a los visitantes.

Esta nueva condición resignifica la relación de los públicos con respecto a la colección; la cual en esta tipología de museo es complejizada al considerar a las piezas como obras de arte. Esta especificidad establece una diferenciación entre los objetos y las obras, resaltando su valor simbólico por su valor de uso en pos de su condición estética; lo que claramente repercute en la manera de ser exhibidas y en la apropiación que se realiza de ellas (Pomian, 1987; Bal, 1996).

Esta manera de identificar a los objetos determinó las colecciones de ciento ocho museos en el territorio argentino. ${ }^{2}$ Sin embargo, estos acervos poseen diferencias en sus atributos que repercute en la identificación de las sedes museales, distinguibles en sus nomenclaturas, particularidades de sus piezas y diferenciación en las actividades de su programaciones, entre otras opciones. Es así que identificamos los museos tradicionales de Bellas Artes, tales como los nacionales en las provincias de Buenos Aires y Neuquén, aquellos de dependencia provincial en Catamarca, Córdoba, Chaco, Corriente, Entre Ríos, Jujuy, La Rioja, Mendoza, Misiones, Salta, Santa Fe, Santiago del Estero y Tucumán, y de orden municipal en distintas ciudades del territorio. Al mismo tiempo, sumamos los museos de arte moderno emplazados en las provincias de Buenos Aires y Mendoza y, los actuales museos de arte contemporáneo en las ciudades de Salta (Salta), Mar del Plata

1 Establecemos tres momentos claves, el primero con el Museo de Louvre que construye el modelo museístico moderno, el segundo con la creación del Centro Pompiu que genera el modelo museístico posmoderno y el tercer modelo museístico contemporáneo a partir de Museo de Guggenin en Bilbao.

2 Según la segunda edición de la Guía Nacional de Museos (año 2013) llevada adelante por el Ministerio de Nación.
(Buenos Aires), Bahía Blanca (Buenos Aires) y Rosario (Santa Fe). Conjuntamente, reconocemos sedes que poseen producciones artísticas específicas: decorativos en Buenos Aires y Santa Fe, oriental en Buenos Aires, y arte popular en Buenos Aires, Entre Ríos, Salta, Santa Fe y Santiago del Estero. Otros que responden a un tipo concreto de producción plástica como pintura naif (Chubut), escultura (Chaco y Misiones) y grabado (Buenos Aires), además de aquellos que se corresponden con piezas de arte sacro ubicados en distintas dependencias de Buenos Aires, Córdoba, Jujuy, La Rioja y Santa Fe. Esta tipificación es completada con los museos/atelier concebidos por colecciones privadas de artistas como Xul Solar (Buenos Aires), Rómulo Raggio (Buenos Aires), Fernando Fader (Mendoza), Lino Enea Spilimbergo (Córdoba) y Francisco Ramoneda (Jujuy).

Todos estos museos, de un modo u otro, han sucumbido a distintas transformaciones para acoplarse a las variaciones del sistema capitalista, generando una versión contemporánea que los resignifica. Entre las modificaciones realizadas distinguimos el acoger y exhibir obras de arte contemporáneo, ${ }^{3}$ abandonar la transmisión de saberes lineales o evolutivos presentados en una exposición anual, exacerbar la lógica de centro cultural, incorporar espacios-servicios de esparcimiento y de consumo, entre otras opciones. Sin embargo, algunos de los cambios que se proyectan en los museos de arte argentinos responden a las lógicas que promulgó el modelo museístico posmoderno, aunque estas a su vez sientan las bases sobre las que se construye el arquetipo contemporáneo. El museo actual se convierte de esta manera no solo en un lugar de aprendizaje, sino también en un espacio de encuentro e interacción con los visitantes, a partir de la relación con las obras o el disfrute de diferentes propuestas que habilita la experiencia museal.

Específicamente, el contacto con el patrimonio durante la visita implica reconocer que cada visitante tiene una vivencia subjetiva única e irrepetible con él; lo cual supone que los museos son experimentados

3 En sintonía con esta lógica se llevan adelante dos claras líneas de prácticas museísticas. Una, vinculada a los establecimientos creados específicamente para albergar y exponer producción contemporánea: el MACSa (Salta), MACRo (Santa Fe), MACU (Buenos Aires) y MAR (Buenos Aires). Y la segunda se relacionada a un grupo de museos tradicionales que adquieren y exhiben obra contemporánea: el Museo "Timoteo Navarro" (Tucumán), el Museo "Emilio Caraffa" (Córdoba), el Museo "Rosa Galisteo" (Santa Fe), el Museo "Juan B. Castagnino" (Santa Fe), el Museo de Arte Moderno (Mendoza), el Museo "René Bruseau" (Chaco), entre otros (Zacharías, 2016). 


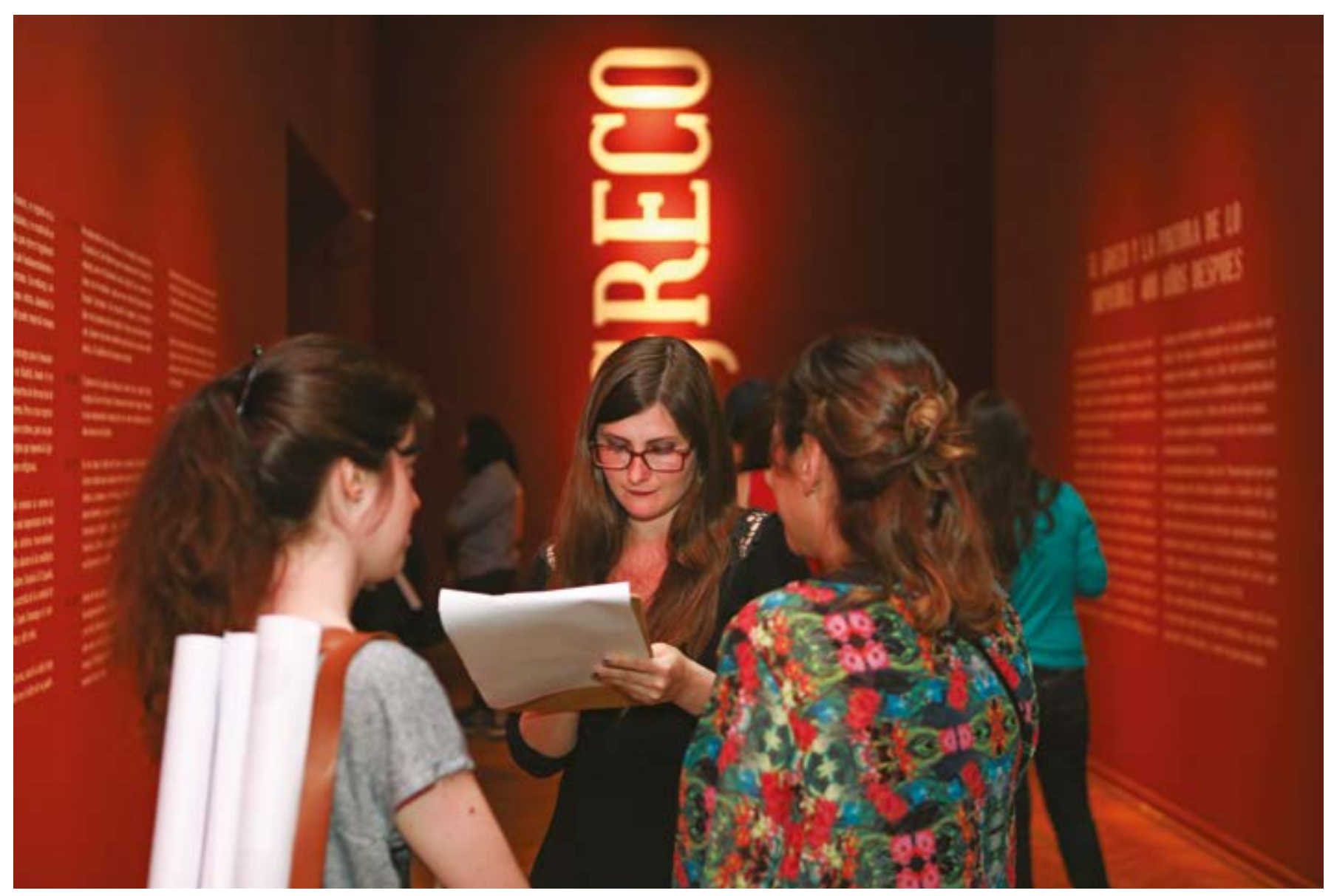

Imagen 1. Museo Nacional de Bellas Artes en el marco de Bellos Jueves 2014, y la exposición temporal El Greco. Cortesía: Matías Iesari / Gustavo Cantoni.

a través de acciones o episodios que realiza el visitante al recorrerlo, participar o disfrutar de las acciones que se construyen con las piezas del acervo. En este caso, al tratarse de museos de arte, es necesario identificar esta experiencia desde lo perceptivo —vinculado a lo "estético"- ${ }^{4}$ lo que implica reconocer la íntima relación que puede provocar en el individuo algunas obras de arte - aunque la experiencia no se reduce solo a ellas-. Estas sensaciones que movilizan la producción artística estimulan la memoria y la reflexión; convirtiéndose así en parte de los procesos sociales. Pero, para lograr este cometido no alcanza con la experiencia que puede suscitar la obra por sí misma; se hace necesario aportar herramientas para que el visitante abandone las condiciones de "receptor pasivo de contenidos y prácticas" y se convierta en un "sujeto activo capaz de

4 Dewey (2008) diferencia lo estético y lo artístico, ya que este último refiere al acto de producción, y por el contrario lo estético responde a la percepción y al goce, el cual se ajusta a la mirada del visitante: "la experiencia consciente como una relación percibida entre el hacer y el padecer, nos capacita para entender la conexión que el arte como producción y percepción, y la apreciación como goce, sostienen recíprocamente." redireccionar aspectos relevantes de las políticas institucionales" (Chagra y Giese, 2015, p. 5).

Este rasgo enmarca la verdadera transformación de los museos del siglo XXI, es decir, es necesario que los mismos desarrollen, visibilicen y profundicen distintas instancias de diálogo o participación entre los visitantes y el patrimonio artístico. Los visitantes, así, se convierten en parte esencial de la experiencia museal, al cobrar voz y co-construir conocimiento sobre las posibles narrativas que visibilizan el arte, otorgando a la entidad, asimismo, la condición de convertirse en verdadero agente inclusivo y democrático.

\section{II.}

Los museos de arte del territorio argentino en las últimas décadas sufrieron transformaciones sustanciales en sus colecciones, fisonomías, puestas expositivas, maneras de comunicar, entre otros cambios. Elena Olivera especifica que se desarrolló una extensa bibliografía sobre algunos de los puntos señalados, pero 


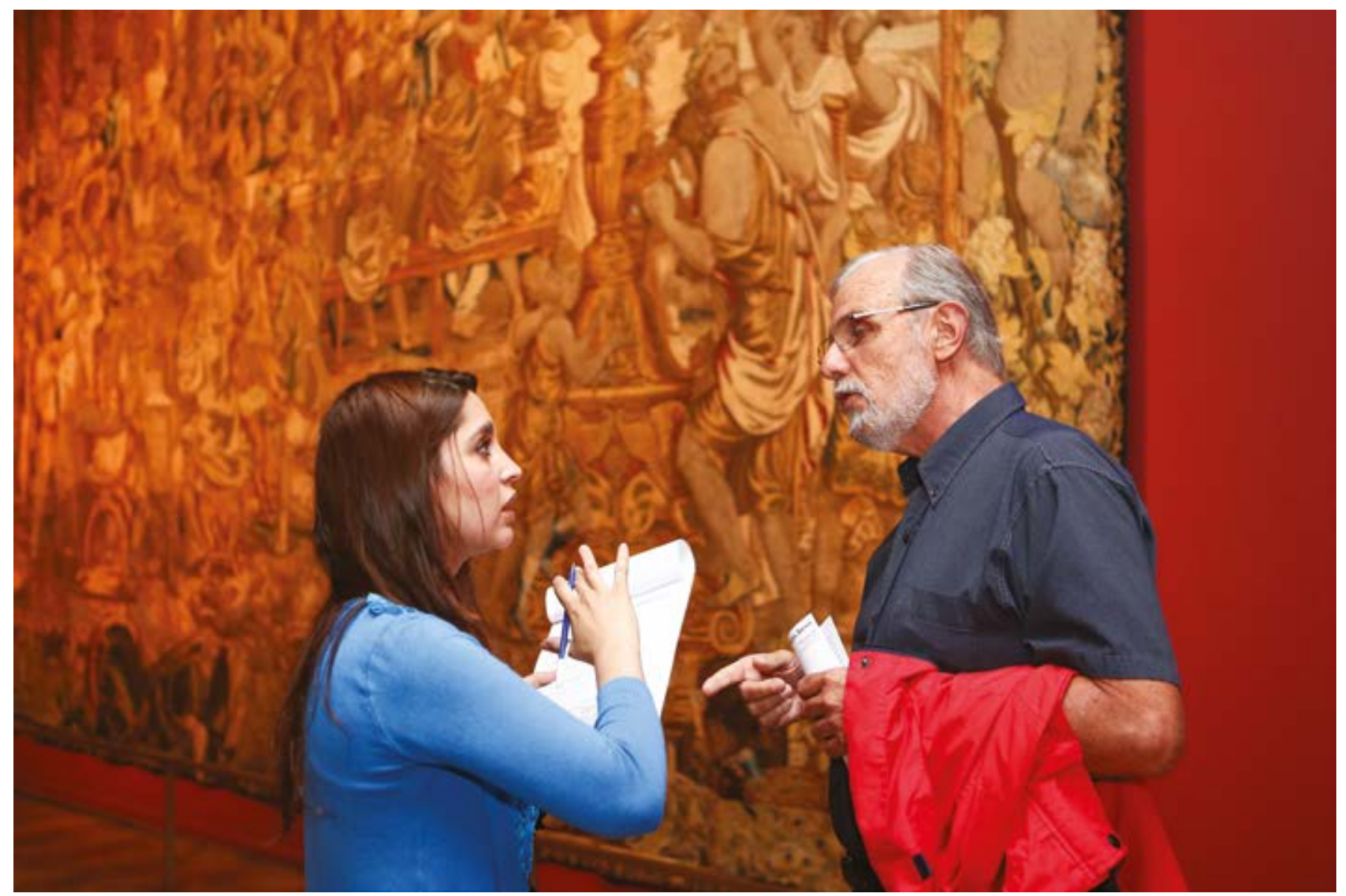

Imagen 2. Museo Nacional de Bellas Artes en el marco de Bellos Jueves 2014, y la exposición temporal El Greco. Cortesía: Matías Iesari / Gustavo Cantoni.

considera que aún son escasos los trabajos que operan en Argentina sobre los visitantes. Por esta razón, reflexiona que "el elemento menos conocido del arte ha sido y sigue siendo su público" (2008, p. 123). En línea con esta afirmación, consideramos que para generar un verdadero cambio con respecto al nuevo rol que tienen que tener los visitantes en los museos, primeramente, es necesario indagar en sus necesidades, gustos, intereses, entre otros.

A modo de ejemplo, traemos a colación la investigación llevada adelante por Pierre Bourdieu y Alain Dardel (2002), quienes profundizaron en el consumo y comportamiento de los visitantes de los museos de arte de París (Francia). En esta exploración llegaron a la conclusión que este tipo de entidad era visitada por un conjunto de individuos que comparte un mismo sistema valorativo y ciertos gustos, los cuales son modelados en relación a sus contextos sociales. Esta referencia se relaciona con que el público (en singular) de estos museos es identificado con un visitante cautivo que posee "una entrada facultativa, reservada a quienes, provistos de la facultad de apropiarse de las obras, tienen el privilegio de utilizar esta libertad y se encuentran de ese modo legitimados en su privilegio" (2002, p. 177). Es decir, a lo largo del tiempo, se considera que esta tipología de museo está reservada para una élite. Olivera también reflexiona en torno a este aspecto y lo conceptualiza como el público de arte, "un tipo de público especializado que debe cumplir al menos con un requisito elemental: la conciencia de que eso que se le enfrenta es, efectivamente, una obra de arte" (2008, p. 124).

Sin embargo, actualmente, esta deferencia sobre el visitante modelo es puesta en tensión a partir de la exhibición de arte contemporáneo. Esta condición repercute, por un lado, en las diferencias de las producciones artísticas $y$, por otro, por los intereses que guían a este actor al recorrer las exposiciones estéticas. Andrea Giunta (2004) distingue un primer grupo que se ciñe a los criterios de juzgar las obras desde la mimesis y el oficio. Estos espectadores destacan la obra por el capital del artista que representa con fidelidad lo que se propone, además de poseer una habilidad en el uso de materiales y técnicas. Por el contrario, el segundo grupo está compuesto por sujetos inmersos en el arte 
contemporáneo (artistas, curadores, historiadores), especialistas — según la autora- que diversificaron sus instrumentos de análisis y se vuelven inmunes a las rarezas de la producción contemporánea. Esta diferenciación se amplía a partir de la clasificación de Guido Ballo (1966), quien plantea una mayor diversidad de visitantes identificados como el ojo común, el snob, el absolutista y el crítico. El primero, identifica la producción contemporánea a partir de eso lo hace cualquiera, eso lo hace mi hijo, el arte hoy es un chiste, en arte todo vale, entre otros. Por esta razón, para este individuo muchas de las obras de arte contemporáneo pasan a ser solo la ocasión para un escándalo público, por tanto, solo se identifica con prácticas y técnicas que respondan a lo tradicional —-mimesis y arte auráticoEl segundo caso, busca sentirse cerca del arte y mostrarse como conocedor de aquello que está en la cresta de la ola, rechazando lo pasado, de modo que solo establece un contacto superficial y sumamente variable según cada propuesta artística. El tercer actor se caracteriza por defender una sola tendencia, lo que no le permite ver más allá de su propio gusto. Por último, el cuarto visitante proyecta un modelo desprejuiciado y abierto a todo lo contemplativo y teórico.

Estos grupos específicos constituyen los posibles visitantes de los museos de arte, pero además permiten identificar a este destinatario no desde un solo registro espectatorial, aunque todos están relacionados de una $u$ otra manera con lo estético. La realidad es que todos ellos presentan diferencias $y$, efectivamente, presentan por lo menos dos o cuatro imaginarios sobre lo artístico. En esta línea, cabe señalar el crecimiento de la visita por parte de aquellos individuos que no están estrechamente vinculados al arte, sino que disfrutan de sus ofertas como un fenómeno de masas. Esta corriente de público (allegado, paseante o mirón) no es identificada como el público del arte, sino que pertenecen a la categoría de turista cultural. Este actor diferente al ojo crítico se desplaza hacia las instituciones culturales por la promoción comercial, es decir, se ve atraído por las nuevas lógicas del ser museístico contemporáneo, y lo consume como un espacio vinculado a la recreación y el ocio. Transformándolo, tal como dice Andrea Huyssen, en la Quinta Avenida en hora pico, en donde "el museo se traduce en la invisibilidad de aquello que se ha ido a ver, esta nueva invisibilidad del arte como forma hasta ahora última de lo sublime" (2007, pp. 56-57).

Estas diferencias que se establecen entre los visitantes, presentan una multiplicidad de regímenes espectatoriales, variedades de consumo y diferentes modos de apreciar las obras de las colecciones; lo cual conlleva distintas maneras de construcción de sentido del discurso museal sobre lo artístico. Ante este abanico de posibilidades, tal como plantea Olivera, se presentan múltiples dificultades, entre las que destaca, el comunicar -el arte es para todos - y el problematizar. “En el primer caso se integra, se reconocen las capacidades de unos y de otros; en el segundo, se dificulta el contacto, se sorprende y se desconcierta al espectador invitándoselo a ver y a construir un sensorium diferente" (2008, p. 126).

Por esta razón, para lograr una entidad democrática es necesario conocer a todos los visitantes y, para ello, es esencial el uso de los estudios de visitantes o públicos. ${ }^{5}$ Estos tipos de búsquedas son un recurso valioso para repensar el vínculo museo-visitantes. Tal como expresa Eilean Hooper-Greenhill (2006) se trata de un conjunto de investigaciones y evaluaciones que involucran a los museos y sus públicos actuales, potenciales o virtuales; las cuales son realizadas con diferentes propósitos por personal de los establecimientos o actores externos. Estas indagaciones se convierten en una herramienta que posibilita medir desde características simples y clásicas (socio-demográficas) hasta aspectos más complejos que buscan detectar gustos, necesidades, expectativas o experiencias de estos actores en el ámbito museal o fuera del mismo. Los resultados que arrojan este tipo de exploraciones permiten a los establecimientos, entre otras posibilidades, resignificar los mensajes de las puestas museográficas, mejorar los servicios ofrecidos, diseñar políticas de acción, planificar actividades específicas, co-construir relatos sobre sus colecciones, es decir, habilitar la voz del visitante en la lógica museal.

Asimismo, estas investigaciones plantean la recepción en términos plurales, lo que implica abandonar la concepción singular de un público, por los públicos. Es así que se identifica una multiplicidad de imaginarios para establecer distintos niveles de interacción o de diálogo con aquello que ofrece cada museo. De esta manera, podemos pensar, por ejemplo, que cada visitante de un museo de arte tiene distintas emociones frente a una obra, se detiene o continua ante la lectura de las cartelas, interactúa o guarda silencio frente a otros, por citar

5 "Los estudios de visitantes o estudios de públicos suelen ser trabajados indistintamente en la bibliografía hispano-hablante. Por el contrario, en los textos de habla inglesa se dividen bajo las terminologías visitor studies o visitor research, que suelen relacionarse con el término audience development" (Pérez Castellano, 2016, p. 23). 


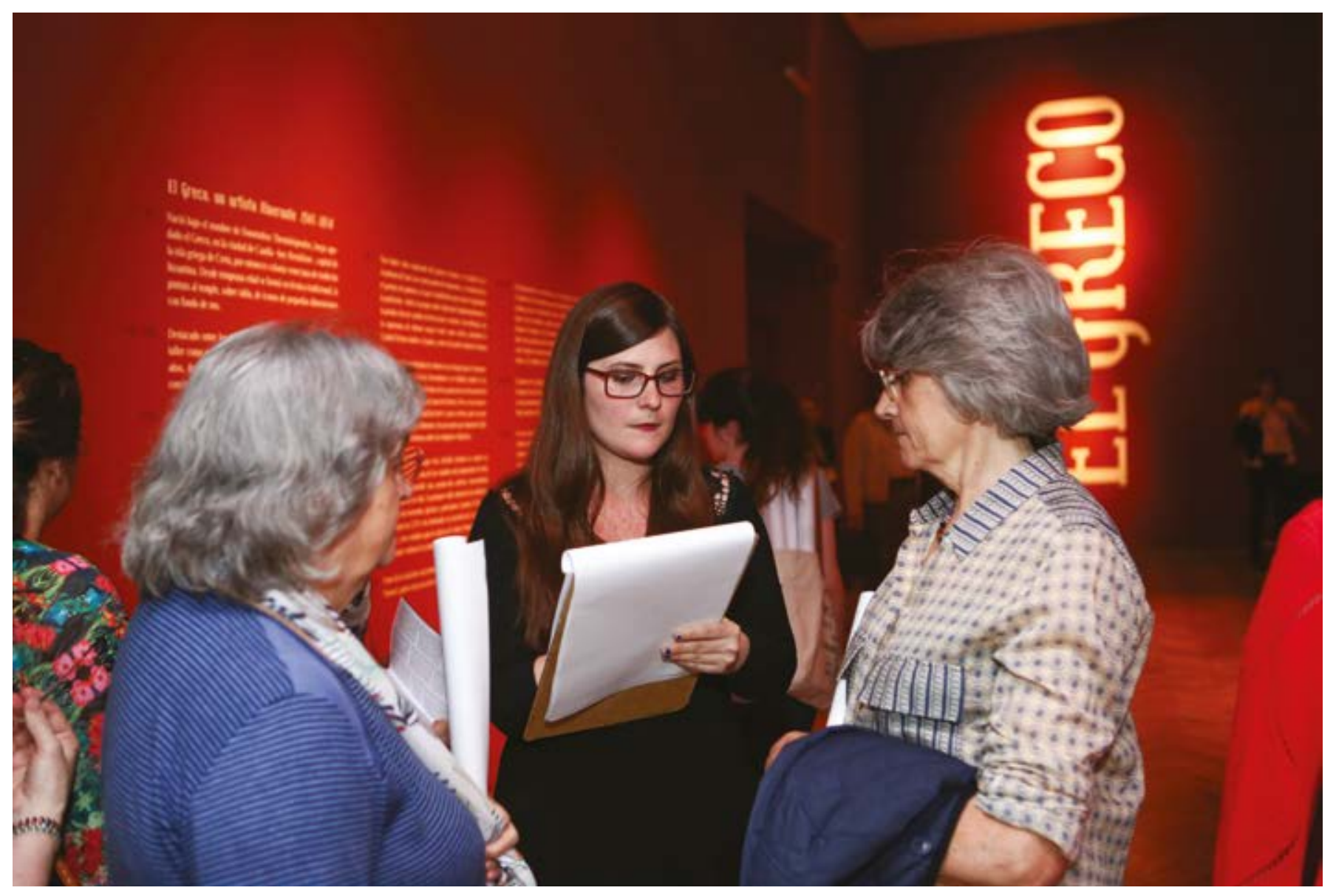

Imagen 3. Museo Nacional de Bellas Artes en el marco de Bellos Jueves 2014, y la exposición temporal El Greco. Cortesía: Matías Iesari / Gustavo Cantoni.

algunas posibilidades. Por ello, conocer a los visitantes proyecta una museología democrática, accesible y plural que habilita establecer la curva de concurrencia, conocer y comprender a los visitantes, satisfacer a los usuarios, son tres cuestiones que hablan de los distintos problemas, saberes y sistemas de acciones que en conjunto determinan la política de público del museo contemporáneo" (Eidelman, Roustan y Goldstein, 2013, p. 21).

No obstante, este tipo de estudios presentan distintas posibilidades según el orden con el que se trabaje, es decir, existen diferencias entre los métodos cuantitativos y cualitativos; lo que obliga a reflexionar sobre el alcance que permite cada uno de ellos. Es decir, no cuestionamos la metodología en relación a los objetivos que se propone cada establecimiento, solo señalamos que la información que proporciona lo cuantitativo son datos puramente estadísticos. Por el contrario, las indagaciones cualitativas establecen múltiples instancias sobre los regímenes espectatoriales y de consumo que ayudan a reconocer una pluralidad de imaginarios y de percepciones acerca de las actitudes, gustos, necesidades e intereses de los visitantes.
A raíz de lo expuesto, si un museo quiere conocer a sus visitantes debe sumar información de ambos métodos. Esta conjunción aborda una serie de variables, no solo externas - contexto físico y social- sino también internas, subjetivas, individuales y únicas de cada individuo. Falk y Diercking (1992) sostiene que este tipo de análisis amplía los márgenes con respecto a los anteriores acercamientos que solo ofrecían datos sobre las características socio-demográficas, los hábitos de visita, usos y gratificaciones de los servicios, entre otros. Un ejemplo de este tipo de investigación mixta fue llevado adelante por Verón y Levasseur en 1983 en el Museo Pompidou. En la misma se identificaron los procesos de reconocimiento por parte de los visitantes al concurrir a la muestra "Vacaciones en Francia 18601982". A partir de los resultados obtenidos pudieron identificar no solo quienes concurrían, sino también los distintos regímenes espectatoriales de cada visitante; lo cual le permitió a la entidad reconstruir el espacio expositivo para futuras exposiciones y mejorar la experiencia estética (Verón y Levasseur, 1983). 
Sin embargo, este tipo de investigación en el territorio argentino no es frecuente, ya sea por la falta de recursos humanos y financieros específicos, prolongados períodos de trabajo o encontrarse con resultados que afecten el desempeño, entre otros. Así pues, advertimos que la adaptación de los museos de arte al principal rasgo con que opera el arquetipo contemporáneo - esto es, alinearse a las necesidades del visitanteaún presenta resistencias. Conocer a los visitantes para poder generar instancias de diálogo o participación sobre lo artístico pone en crisis los cimientos sobre los que se construyó esta entidad -autoreferencialidad y autojustificación-, y los obliga a compartir el poder de decisión de las dinámicas museales.

\section{III.}

El primer estudio de público realizado por un espacio artístico en el territorio argentino data de 1964, dirigido por Regina Gibaja, con el título “El público del arte". El trabajo fue encargado por el Instituto Torcuato di Tella al Instituto de Sociología de la Universidad Nacional de Buenos Aires. El mismo consistió en una encuesta aplicada a los visitantes de una exposición de arte para indagar diversos aspectos sociodemográficos, educativos y profesionales, así como también las actitudes y valoraciones de la cultura, el arte y los medios de comunicación. Sin embargo, este ejemplo se trata de un hecho aislado, ya que los estudios de visitantes comienzan a cobrar impulso en Argentina, al igual que en otros países de América Latina, con la vuelta del sistema democrático. Este último impulsó una renovación de las instituciones culturales y, para ello, se valió de las herramientas que aportaban estas indagaciones para conocer a los visitantes. No obstante, en esos años, Vanina Papalini y Marina Moguillansky arguyen que los análisis "se basaron en metodologías cuantitativas, casi siempre a partir de encuestas a los visitantes" (2016, p. 95).

Al no existir datos específicos sobre el uso de los estudios de público en los museos de arte, salvo indagaciones generales de la entidad Ibermuseos realizadas específicamente a museos de carácter nacional (2014) y la investigación de Paola Fritz (2017) que aporta un registro de algunos establecimientos museísticos y sus modalidades de aplicación, se envió en octubre de 2017 un breve cuestionario a los museos de arte argentinos para consultarlos sobre si en alguna ocasión realizaron estudios de visitantes, sin distinguir entre entidades públicas o privadas. ${ }^{6}$ Del total encuestado 7 solo aportaron información treinta y cuatro entidades, las cuales representan un $34,4 \%$ del total. A pesar de que el tamaño de la muestra es escueto, marca un primer acercamiento al estado de situación en el uso de esta herramienta y, principalmente, permite identificar algunas indagaciones específicas para repensar y reconstruir aspectos vinculados a la experiencia museal y estética.

Del análisis realizado identificamos que siete entidades $^{8}$ no realizaron en ningún momento estudios de visitantes. En cuanto a las que sí utilizaron este tipo de herramienta, solo diez $z^{9}$ trabajaron con metodología cuantitativa y doce aplicaron recursos de ambos métodos - cualitativos y cuantitativos-. Específicamente, sobre este último grupo identificamos tres museos nacionales ${ }^{10}$ — sus exploraciones son llevadas adelante por el Ministerio de Cultura de la Nación-y tres de

6 Del total de entidades, ochenta y tres son de carácter público, veinticuatro responden al orden privado y solo tres poseen condición mixta. La diferencia en estos números se debe a que en el territorio argentino la mayoría de sus museos se sostienen bajo la concepción y creación que trazan los lineamientos planteados por las entidades museísticas europeas. Si bien las condiciones administrativas del Estado Argentino, según Raúl D'Amelio (2008) presentan diferencias respecto a algunos países europeos, encontrando tres niveles de procedencia: nacional, provincial y municipal o comunal. Además, de este paisaje institucional no queremos dejar de mencionar que tres sedes museales son de carácter universitario. Esta condición a su vez los ubica dentro de aquellos de carácter público, ya que las Universidades Nacionales de Lomas de Zamora, de Cuyo y del Litoral, son entes autárquicos, pero con dependencia del Estado Nacional Argentino.

$7 \quad$ Es de señalar que del total se desestimaron siete entidades que no cumplen con el requisito de tener una colección y espacio físico delimitado.

8 Museo Universitario de Arte de UNCUYO (Mendoza), Museo de Arte "Eduardo Minnicelli" (Santa Cruz), Museo Municipal de Artes Visuales "Sor Josefa Díaz y Clucellas" (Santa Fe), Museo Bellas Artes "Laureano Brizuela" (Catamarca), Museo de Bellas Artes de Tres Arroyos (Buenos Aires), Museo de Bellas Artes de Bahía Blanca (Buenos Aires) y Museo de Arte Contemporáneo de Bahía Blanca (Buenos Aires).

9 Museo Pictórico "Luis José Pisano" (Córdoba), Museo Bellas Artes "Laureano Brizuela" (Catamarca), Museo de Arte Contemporáneo de Salta (Salta), Museo de Arte Moderno (CABABuenos Aires), Museo Provincial de Bellas Artes "Timoteo Navarro" (Tucumán), Museo de Arte "Juan de Tejeda" (Córdoba), Museo Provincial de Bellas Artes "Rosa Galisteo" (Santa Fe), Museo de Arte de Tigre (Buenos Aires), Museo de Arte Latinoamericano de La Plata (Buenos Aires) y Museo de Arte Sacro de Rosario (Santa Fe).

10 Museo Nacional de Arte Decorativo, Museo Nacional de Arte Oriental y Museo Nacional de Grabado. 
carácter municipal (Rosario-Santa Fe) ${ }^{11}$ son llevadas adelante por la Secretaría de Cultura y Educación y la Dirección de Museos de Rosario. En ambos casos, se tratan de cuestionarios que recolectan datos demográficos y algunas preguntas de apreciación sobre el patrimonio y lo edilicio. De las entidades restantes (seis) queremos destacar que efectuaron indagaciones — sistematizadas en el tiempo o en casos puntuales-, en donde identificamos la percepción de los visitantes a partir de recorridos o actividades generadas con algunas piezas de la colección. Este tipo de relevamiento en mayor o menor medida permitió a estos museos resignificar narrativas, servicios, ofertas, entre otras posibilidades.

Una de las sedes museales que llevó a cabo una investigación mixta fue el Museo Nacional de Bellas Artes (Buenos Aires), dependiente del Ministerio de Cultura de la Nación, que realizó estudios de manera sistemática e independiente desde el año 2013. Entre sus indagaciones -cuestionarios semi-estructurado y libros de visitas - destacamos las exploraciones a las exhibiciones permanentes y temporales. Específicamente, se relevaron los niveles de satisfacción respecto de aquello que el visitante necesita para ser más cómoda su estadía, y la nueva propuesta curatorial de las salas de arte del siglo XX. Los resultados coincidieron con un alto nivel de satisfacción, y ante algunas sugerencias sobre el mantenimiento de las instalaciones u otras, los reclamos comenzaron a ser atendidos al ser también detectados en otros estudios (Chagra y Giese, 2015).

En segundo lugar, ubicamos el Museo Municipal de Arte "Julio Pagano" de Rafaela (Santa Fe); el cual desarrolló un acercamiento a las producciones plásticas de los primeros artistas locales para reconstituir el patrimonio artístico. Para ello, apelaron a entrevistas con diferentes instituciones públicas y privadas, como también con los familiares de los creadores plásticos. Además, trabajaron junto con los alumnos de las escuelas -a través de breves cuestionarios - la identificación de aspectos sobre lo patrimonial —qué tipo de información brindar y qué obras o temáticas seleccionar- que fueron incorporados en el recorrido expositivo. De este resultado se generó una investigación específica sobre la colección, la adquisición de nuevas obras y la puesta museográfica de una exposición patrimonial.
El siguiente caso corresponde al Museo de Arte Étnico Americano-Pajcha (Salta) de carácter privado. El estudio fue llevado a cabo por miembros de la entidad, junto con la licenciada chilena Olaya Sanfuentes, para trabajar el cúltico popular a partir de las imágenes de San Santiago (Apóstol Ecuestre) -delimitado en un sector de exposición-con el público local, descendientes de las culturas precoloniales y los visitantes extranjeros (Sanfuentes y Ossa, 2017). Posteriormente, el estudio habilitó a la entidad a la reconstrucción de ese sector del espacio expositivo y generó una publicación en torno a la relación estética y el culto religioso, además de las subjetividades que despierta esta figura religiosa.

El cuarto caso se trata de la entidad privada, Museo de Arte Latinoamericano de Buenos Aires (MALBA); el cual realizó distintos estudios, entre ellos la exploración a la exposición Verboamérica. Esta indagación fue impulsada por su área de Educación, más específicamente, por Gabriela Santagostino (referente interno) y la colaboración de Florencia Romera (investigadora invitada). La investigación se apoyó en el relevamiento del espacio, encuestas, entrevistas, observaciones en sala, análisis de la comunicación externa e interna, con el foco puesto en el análisis de la experiencia de la visita a dicha exposición. Los resultados fueron tenidos en cuenta para la reconstrucción del guión de las visitas guiadas que se realizaron posteriormente y para futuras mediaciones.

El caso número cinco refiere al Museo Superior de Bellas Artes Evita-Palacio Ferreyra, en el cual hace aproximadamente dos años un grupo del taller de adultos mayores realizó un cuestionario en el que se identificó que las personas que visitaban el museo querían tener información sobre la edificación y la familia que lo construyó. Por esta razón, se autorizó, a fines del año 2017, a este grupo junto con personal del museo, construir una exposición en una de las salas del piso inferior que diera cuenta de algunos de estos aspectos en su narrativa.

Por último, el caso más interesante de todas las propuestas relevadas es el Museo de Arte Popular "José Hernández", de orden público, dependiente de la Dirección General de Patrimonio, Museos y Casco Histórico de Capital Federal (Buenos Aires). Desde 2001
11 Museo de Arte Contemporáneo de Rosario, Museo de Bellas Artes "Juan B. Castagnino" y Museo de Artes Decorativas "Emilia y Odilio Estévez". 


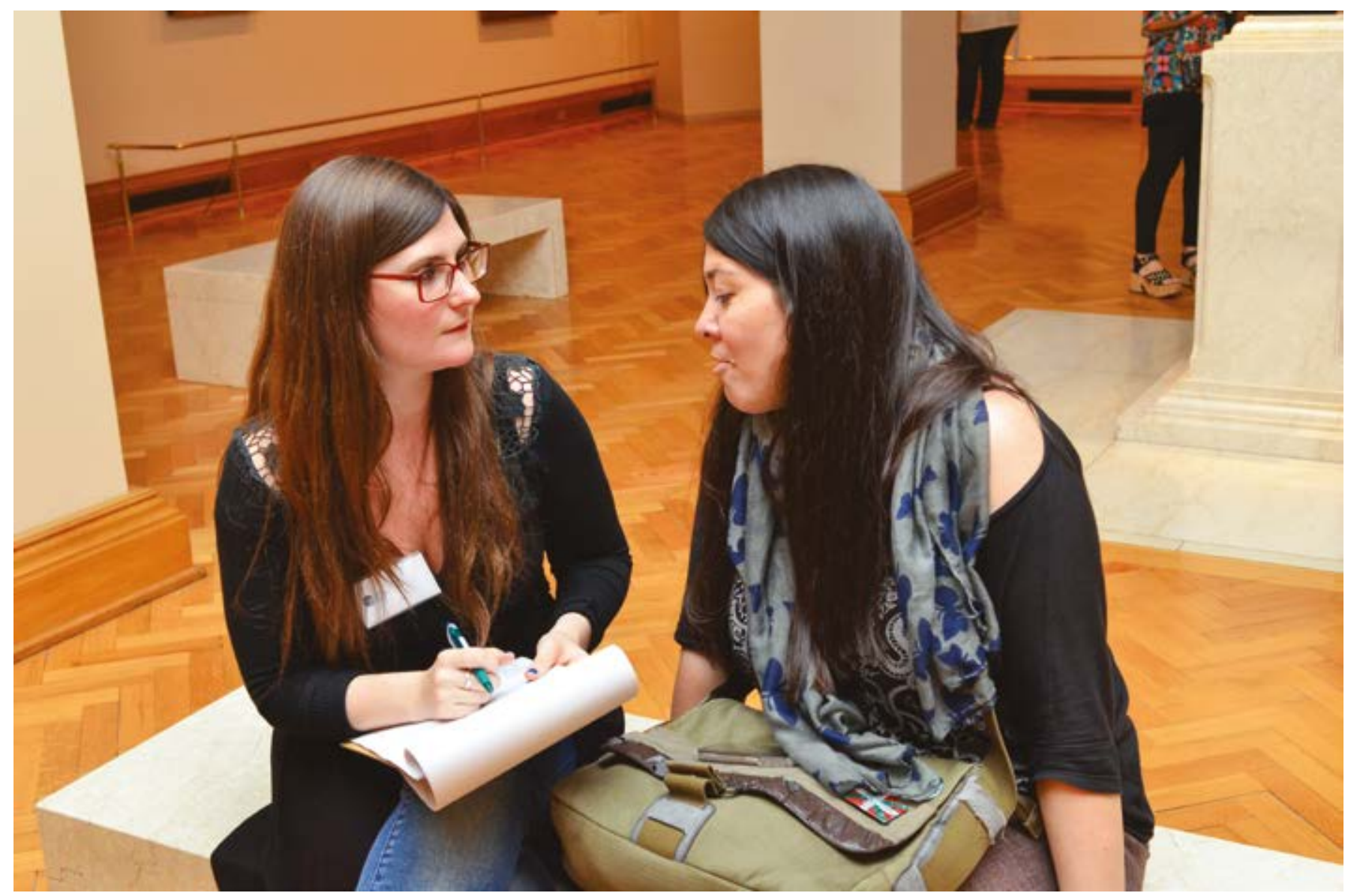

Imagen 4. Museo Nacional de Bellas Artes en el marco de Bellos Jueves 2014, y la exposición temporal El Greco. Cortesía: Matías Iesari / Gustavo Cantoni.

—continúa en la actualidad - ${ }^{12}$ delineó un programa de estudios de público de tipo cualitativo por medio de entrevistas abiertas, con el fin de indagar exposiciones particulares y el Museo Hernández en general. Estos estudios registraban, por un lado, las demandas y las valoraciones de los visitantes $y$, por otro, monitoreaban la eficacia comunicativa de sus propuestas. De las distintas exploraciones destacamos dos, ya que permitieron transformar la lógica del museo y su colección. La primera fue la decisión del cambio de imagen institucional para identificar al Museo Hernández como el Museo

12 Anteriormente, se realizaron actividades en esta línea, en el año 1997, a través de un convenio entre la Dirección del Museo de Arte Popular José Hernández, y el Instituto de Ciencias Antropológicas de la Facultad de Filosofía y Letras de la Universidad de Buenos Aires, se llevó a cabo la primera pasantía de Estudios de Público, experiencia que se reiteró en 1998. También ese año, la Lic. Ana M. Cousillas, directora de la institución, dictó un seminario de grado en dicha universidad acerca de la investigación de la interpretación del patrimonio cultural en los museos (Cousillas, 1997). Igualmente, organizó el Primer Seminario Internacional denominado "Estudios de Visitantes a Museos y Evaluación de Exposiciones", que convocó a diferentes especialistas en la materia. de arte popular. La medida se debió a que se identificó que los visitantes recurrían a interpretar el patrimonio -representativo de la identidad nacional- asociado a lo gauchesco, lo telúrico, lo del interior, sin referenciar la presencia indígena y la población inmigratoria del país, a pesar de que ambas estaban representadas en la muestra permanente y en la cartelería (Bialogorski y Cousillas, 2000; 2003). ${ }^{13}$ Atendiendo a sus imaginarios se construyeron nuevas propuestas comunicativas y museográficas que resignificaron la interpretación del acervo, lo cual transformó el discurso que llegaba a los visitantes. La segunda disposición se basó en un estudio con base semiótica que intentó definir la construcción simbólica de la interpretación del patrimonio del Museo Hernández y del campo artesanal. Es así como se indagó acerca de conceptos tales como artesanía, artesanía tradicional, artesanía urbana, arte popular. Las construcciones realizadas de estos tópicos por parte del

13 Una visión que concordaba con el mensaje claramente criollista que había adoptado la institución a partir de 1955, y que se mantuvo con variantes hasta los años 80 fuertemente fijada en el imaginario porteño. 
público dieron lugar a distintas propuestas para la confección de la programación. (Bialogorki y Fritz, 2012).

\section{IV.}

A pesar de los pocos datos generales que dimos cuenta a lo largo del texto, identificamos que en los últimos años se percibe una mayor utilización de los estudios de visitantes por parte de los museos de arte argentinos, aunque bajo dos líneas específicas de trabajo. Por un lado, un grupo de indagaciones que recaban mayoritariamente información socio-cultural, que en muchos casos solo genera datos parciales y circunstanciales, sin precisiones metodológicas que posibiliten establecer su rigor. Además, estos carecen de continuidad para lograr ir más allá de la fotografía de un momento específico, no permitiendo establecer tendencias a mediano o largo plazo. Por otro lado, destacamos que en los casos descritos - si bien algunos de ellos son incipientes o profundizan aspectos puntuales - en el último apartado se detecta el uso de metodologías de orden cuantitativo y cualitativo que permiten detectar los intereses, gustos y necesidades de sus visitantes para luego generar nuevas propuestas en su programación, espacios-servicios, narrativas, entre otras posibilidades.

A modo de cierre, reconocemos que el museo contemporáneo aún está en construcción, porque la contemporaneidad se está pensando día a día, en la propia dinámica del presente. Dicha dinámica implica una nueva estructura comunicacional abierta al diálogo y al rol activo de los visitantes que pone el acento en nuevos mecanismos complejos de circulación de sentido que conllevan nuevas lógicas sobre el hacer de este tipo de institución cultural. Creemos, sin embargo, que en los museos de arte lo artístico continúa siendo abordado principalmente desde la lógica del objeto (colección) o desde el público del arte. Todavía queda mucho camino por recorrer para que el museo se trate de una alternativa a la lógica que plantean algunas instituciones culturales establecidas, un complemento para generar y construir nuevas dimensiones de lo museal.

\section{Referencias}

Bailo, G. (1966). Occhio Critico. II nuovo sistema per vedere larte. Milano: Longanesi.
Bal, M. (1996). Double Exposures. The subject of cultural analysis, in R. Greenberg (Ed.), Thinking about Exhibitions, pp. 201-218. London: Routledge.

Bennett, T. (1996). “The exhibitionary complex”, in T. Prasch (Ed.) Thinking about Exhibitions, pp. 69-92. London: Routledge.

Bialogoski, M. y Cousillas, A. M. (2000). “Gestión cultural y estudios de Público en el Museo José Hernández", en Cuadernos de Antropología Social (12), pp. 197-205, Buenos Aires.

(1980). The Universal Survey Museum, in Art History, 3, pp. 447-469. Nueva York. . (2003). Estereotipos y preconceptos en la interpretación del patrimonio cultural en el Museo José Hernández. Buenos Aires: Ediciones del Instituto Nacional del Arte (IUNA).

Bourdieu, P. y Darbel, A. (2002). El amor al arte. Los museos europeos y su público. Buenos Aires: Paidós.

Chagra, N. y Giese, A. I. (2015). ¿Para quién es el museo? Buenos Aires: Museo Nacional de Bellas Artes.

Cousillas, A. M. (1997). Guía sobre estudios de visitantes a museos. Buenos Aires: INAPP.

D'Amelio, R. (2008). “Encuentro con los museos de Rosario (Argentina)", en Revista museos.es, 4, pp. $170-$ 181. Madrid: Ministerio de Educación, Cultura y Deportes.

Dewey, J. (2008). El arte como experiencia. Barcelona: Paidós.

Duncan, C. (1995). Civilizing Rituals. Inside Public Art Museums. Nueva York: Routledge.

Duncan, C. y Wallach, A. (1978). The Museum of Modern Art as Late Capitalist Ritual, in Marxist Perspectives 1(4), pp. 28-51. Winter (EEUU).

(2012). “Diálogo con los visitantes. Estudio de Público cualitativos en el Museo de Arte Popular José Hernández de la Ciudad de Buenos Aires", en Actas del Simposio Internacional de Museología: Nuevas Prácticas, Nuevas audiencias. A 40 años de la Mesa de Santiago, Santiago de Chile, Chile. 
Eidelman, J.; Roustan, M. y Goldstein, B. (2013). El museo y sus públicos. El visitante tiene la palabra. Buenos Aires: Ariel.

Falk, J. H. y Diercking, L. (1992). The Museum Experience. Washington: Whalesback Books.

Fritz, P. (2017). "Una primera aproximación a los estudios de público en Argentina", en Museos y visitantes: ensayos sobre estudios de público en Argentina, coordinado por M. Bialogorski y M. M. Reca, pp. 181-205. Bs. As: ICOM Argentina.

Giunta, A. (2004). "Vanguardias y arte contemporáneo", en Arte Argentino Contemporáneo, pp. 15-21. Rosario: Ediciones Macro.

Hooper-Greenhill, E. (2006). Studying visitors. Companion to Museum Studies. Londres: Blackwell Publishing.

Huyssen, A. (2007). "Escapar de la amnesia: los museos como medios de masas", en En busca del futuro perdido Cultura y memoria en tiempo de globalización, dirigido por A. Huyssen, pp. 41-73. Buenos Aires: Fondo de Cultura Económica.

Ibermuseos. (2014). Estudios de Público de Museos en Iberoamérica. Observatorio Iberoamericano de Museos (OIM). Madrid: Programa Ibermuseos.
Olivera, E. (2008). Cuestiones De Arte Contemporáneo. Hacia un nuevo espectador en el siglo XXI. Buenos Aires: EMECÉ.

Papalina, V. y Moguillansky, M. (2016). "Los estudios sobre los públicos de Artes", en Estudios sobre consumos culturales en la Argentina contemporánea. Coordinado por M. Grillo (Ed.), pp. 129-175, Buenos Aires: CLACSO.

Pérez Castellano, L. (2016). Estudios sobre públicos y museos. México: Publicaciones ENCRyM.

Pomian, K. (1987). Collectionneurs, amateur's etcurieux. Paris: Gallimard.

Sanfuentes, O. y Ossa, B. (2017). From the Feast Day in Belén to the Museum in Salta: Three-Dimensional Images of St. James the Apostle in Two Different Contexts, in Material Religion. The Journal of Objects, Art and Belief, 13, pp. 52-76. USA.

Verón, E. y Levasseur, M. (1984). L'espace, le corps et le sens. Ethnograpihe de l'exposiction. Paris: Bibliothèque Publique d'Information- Centre Georges Pompidou.

Zacharias, M. P. (2016). Estado del arte. Buenos Aires: Ministerio de la Nación. 